\title{
ASPECTOS ORGANIZATIVOS Y TÉCNICOS PARA LA INTEGRACIÓN DE TABLETAS DIGITALES EN CENTROS DE EDUCACIÓN PRIMARIA Y SECUNDARIA DE CATALUÑA
}

\author{
ORGANIZATIONAL AND TECHNICAL ISSUES FOR THE \\ INTEGRATION OF TABLETS IN ELEMENTARY AND SECONDARY \\ SCHOOLS OF CATALONIA
}

Laura Fernández Rodrigo; laurafernandez@pip.udl.cat

Xavier Carrera Farran; carrera@pip.udl.cat

Universitat de Lleida

\section{RESUMEN}

El objetivo de esta investigación es describir aspectos organizativos y técnicos que los centros de educación primaria y secundaria de Cataluña han tenido en cuenta para la integración de tabletas digitales. Es un estudio cualitativo y microetnográfico en el que se han realizado 15 entrevistas a equipos directivos, coordinadores TIC y familias de 5 centros participantes. Los resultados muestran que los centros tienen en cuenta la experiencia con las TIC, formación docente, infraestructura de conectividad, mantenimiento, organización para su distribución e información para las familias. Las diferencias y semejanzas en la incorporación radican entre el modelo de 1x1 y los modelos de distribución en los que los dispositivos son propiedad del centro.

PALABRAS CLAVE: tabletas digitales, educación, TIC.

\section{ABSTRACT}

The investigation aims to describe the organizational and technical issues to integrate tablets in elementary and secondary schools in Catalonia. It is a qualitative and microethnographic study, in which 15 interviews are carried out to directors, ICT coordinators and families of the 5 participating centres. The results show that the schools consider the experience with ICT, teacher training, connectivity infrastructure, maintenance, organization for the distribution and information for families. The differences and similarities in the incorporation reside between the $1 \times 1$ model and the distribution models in which the devices are owned by the centre.

KEYWORDS: tablets, education, ITC. 


\section{INTRODUCCIÓN}

A partir del curso 2009-2010 el "Departament d'Ensenyament" de Cataluña pone en marcha el proyecto Educat $1 \times 1$ en los centros de secundaria con el fin de que cada alumno pudiera adquirir un ordenador portátil subvencionado. Muchos centros se añaden a este proyecto de inmediato, pero sin las infraestructuras adecuadas, sin una revisión de la programación curricular, sin un replanteamiento del enfoque de la docencia y sin tener experiencias previas (Consell Superior d'Avaluació del Sistema Educatiu, 2010), se dieron una serie de problemáticas en las aulas que hicieron frenar este proyecto, aparte de la inversión económica que suponía para la Generalitat y para los mismos centros. Los centros educativos, a partir del curso 2012-2013, se replantean la forma de continuar con el uso de las TIC en las aulas y muchos se plantean la utilización de otros dispositivos, como la tableta digital (Consorci d’Educació de Barceloma, 2013).

En el año 2013 la Generalitat de Cataluña, conjuntamente con la fundación Mobile World Capital Barcelona, el Ayuntamiento de Barcelona y la GSMA, inician el programa $\mathrm{mSchools.} \mathrm{Es} \mathrm{un} \mathrm{programa} \mathrm{educativo} \mathrm{pionero} \mathrm{destinado} \mathrm{a} \mathrm{los} \mathrm{estudiantes} \mathrm{de} \mathrm{primaria} \mathrm{y}$ secundaria de Cataluña que pretende aprovechar la potencialidad de los dispositivos móviles en el proceso de aprendizaje (Mobile World Capital Barcelona, 2013). Desde esta iniciativa, se reconocen, a través de concursos, las innovaciones didácticas con la finalidad de reducir la fractura digital en las aulas. Se propone realizar visitas escolares al Mobile World Centre, se motiva a los centros para trabajar con el diseño de apps y para diseñar otro tipo de actividades con estos dispositivos.

Con la creación de mSchools, se promueve el uso de tabletas digitales y se suman los centros que quieren aprovechar las funcionalidades de estos dispositivos. Una tableta digital puede servir para múltiples tareas como, por ejemplo, consultar información y documentación, crear material audiovisual, comunicarse a través de redes sociales y compartir material virtual a través de sistemas de almacenamiento en la nube (Cataldi \& Lage, 2013).

\section{OBJETIVO DEL ESTUDIO}

Para la incorporación de las tabletas digitales Vacchieri (2013) expone que "no es evidente que la evolución de los diferentes modelos de integración de TIC en la educación hoy vigentes resulte en un modelo educativo basado en dispositivos móviles" (p. 99 -100), por lo que no existe ningún modelo estándar para todas las escuelas. Con esta idea, el "Departament d'Ensenyament" de Cataluña propone a los centros que elaboren un Plan TAC (Tecnologías para el Aprendizaje y el Conocimiento), que es una estrategia que contempla la manera en que las TIC pueden ayudar en el logro de los objetivos educativos (Fornell \& Vivancos, 2010). De este modo, los centros educativos que deseen incorporar cualquier dispositivo tecnológico deberían de planearse cómo llevar a cabo este proceso.

El objetivo de este trabajo es describir aspectos organizativos y técnicos que los centros de educación primaria y secundaria de Cataluña han tenido en cuenta para la integración de tabletas digitales. 


\section{MARCO TEÓRICO}

\subsection{La integración pedagógica de las TIC}

Cuando se pretende integrar tabletas - u otro dispositivo TIC- en el aula, no sólo hay que mirar de adquirir y acceder a la tecnología, sino de "construir con ellas un modelo pedagógico que atienda a los diferentes niveles de conocimiento, puntos de partida, ritmos de los alumnos y que favorezca el desarrollo de cada sujeto" (Camarda, 2012). El Modelo de Substitución, Aumento, Modificación y Redefinición (SAMR) (Puentedura, 2010; Romrell, Kidder, \& Wood, 2014) consiste en un conjunto jerárquico de 4 niveles que permite evaluar la forma en la cual las tecnologías son utilizadas por los docentes y estudiados des de los centros educativos. La finalidad de este modelo es la de ayudar a los docentes a evaluar el modo en qué se están incorporando las tecnologías a sus aulas, así como conocer qué tipos de usos de la tecnología tienen un mayor o menor efecto sobre el aprendizaje de los estudiantes. Las cuatro fases que componen este modelo están divididas en dos etapas.

En la primera etapa se encuentran las fases a través de las cuales se pretende producir una mejora:

- Sustitución: Es el nivel más bajo de esta escala. Se produce un cambio a nivel instrumental, ya que las TIC se utilizan solo como sustitución de otras herramientas utilizadas.

- Aumento: La nueva herramienta TIC, que ha ocupado el lugar de la anterior proporciona mejoras funcionales que facilitan su tarea, aunque el efecto de los resultados es mínimo o nulo y no se ha dado ningún cambio metodológico.

Después de poder evidenciar la eficacia del nuevo elemento tecnológico, es cuando se empieza a producir una transformación:

- Modificación: En esta fase se produce un cambio metodológico en la cual la tarea a realizar es rediseñada a causa de la introducción de la tecnología. Las actividades que se lleban a cabo cotidianamente se pueden modificar para realizarlas teniendo en cuenta las ventajas del uso de la nueva tecnología.

- Redefinición: Se crean nuevas actividades y ambientes de aprendizaje que serán imposibles de realizar sin el uso de esta tecnología. En este momento, se llega al nivel en el que sin la tecnología sería imposible de realizar la actividad planteada.

\subsection{Modelos para la organización de dispositivos en las aulas}

La finalidad a la que se deberían dirigir todos los centros que quieran incorporar tabletas digitales, según Marquès (Marquès, 2013), es el modelo $1 \times 1$, que significa que en el aula se dispone de una tableta para cada alumno. También se establecen otros modelos de implementación de tabletas, como son los siguientes (Marquès, 2013; Vacchieri, 2013):

- Aula de tabletas digitales o aula TIC: El centro dispone de un aula donde se guardan permanentemente las tabletas y a la cual los alumnos tienen que trasladarse para utilizarlas. 
* Tabletas de soporte en el aula: En cada aula se dispone de un número reducido de tabletas, que se guardan en un espacio determinado y que están disponibles en cualquier momento para los alumnos y docentes de la misma aula.

* Carros móviles de tabletas digitales: En el centro hay un carro adaptado para guardar, trasladar y cargar las baterías de las tabletas. Se establece un espacio para este carro, que esté ubicado en la zona determinada del centro a la cual tiene que dar servicio. Tiene que haber un encargado para organizar una tabla horaria en la cual los maestros y profesores puedan reservar las tabletas necesarias para sus clases.

\subsection{Aspectos técnicos a considerar}

Hugo Martínez, (en Carneiro, Toscano, \& Díaz, 2011) expone tres desafíos que deben ser afrontados para poder integrar las tecnologías en las aulas escolares:

- Diseñar, mantener y gestionar la infraestructura tecnológica, ya que es conveniente que el centro esté preparado para poder asumir la incorporación de los dispositivos.

- Asegurar la competencia digital docente para la integración curricular de las TIC en el centro educativo, a fin de poder emplear estas herramientas en las aulas.

- Provisionar recursos y contenidos digitales que "que favorezcan el uso e integración pedagógica de las capacidades instaladas en el establecimiento" ( $p$. 66).

A partir de las 9 recomendaciones basadas en la puesta en práctica del proyecto internacional "Tablet Pilot" (Balanskat, 2013), hay que tener en cuenta, a la hora de implementar un proyecto piloto de $1 \times 1$ con tabletas digitales, los siguientes aspectos clave:

1) El perfil y el entorno de los docentes y el aprendizaje que se realiza con las TIC.

2) La portabilidad del dispositivo para aprovechar diferentes entornos de aprendizaje, como diferentes espacios del mismo centro o en el exterior.

3) La necesidad por parte de los docentes para experimentar con la tableta digital, haciendo uso del dispositivo a largo plazo como herramienta cotidiana y de comunicación entre docentes, alumnos y familias.

4) El abordaje de temas amplios en la formación continua de todos los docentes que participen en el plan piloto.

5) El establecimiento de una relación entre la formación continua y la práctica cotidiana de aula.

6) La promoción entre los docentes para compartir, de manera colaborativa a través de la tableta digital, recursos y las experiencias documentadas sobre sus prácticas.

7) Los conocimientos previos de los docentes sobre prácticas pedagógicas, con y sin TIC, para ofrecer otros métodos para aplicar. 
8) El suficiente acceso a Internet en el centro y la proporción a los alumnos de los mismos o similares dispositivos que tienen los docentes.

9) La participación en escenarios de aprendizaje pedagógicos, presenciales o no, para apoyar estrategias de aprendizaje con los dispositivos.

\section{METODOLOGÍA}

Con la finalidad de comprender e interpretar los fenómenos de una realidad construida socialmente (Hedberg, 2008), la investigación se enmarca dentro del paradigma interpretativo. Se desarrolla a través de una metodología cualitativa, que se refiere a aquellos procedimientos de investigación que proporcionan datos descriptivos contextualizadas en el ámbito social donde ocurren naturalmente (Bisquerra, 2009). Se utiliza un método microetnográfcio, que consiste en la descripción y interpretación de una situación social concreta (Spradey, 1980).

Como técnica de recogida de información, se ha escogido la entrevista semiestructurada, que se realiza a través de una pauta de preguntas ordenadas y redactadas permitiendo una respuesta abierta y libre (Canales, 2006). A partir de los objetivos planteados para la investigación, se elaboraron 3 guiones de entrevista, con preguntas diferentes, dado que van dirigidas a tres colectivos de cada centro: equipo directivo, coordinador/a TIC y familias. Las preguntas fueron clasificadas por categorías, que son: motivo de la incorporación, aplicación, coordinación entre niveles educativos, gestión de las tabletas en el centro, ventajas, problemas y limitaciones. Los guiones fueron validados por dos expertos, incluyendo una representante del "Departament d'Ensenyament" de Cataluña.

Se escogieron centros educativos de modo intencionado, a través del "Departament d'Ensenyament", teniendo en cuenta que tuvieran más de un curso escolar de experiencia en la implementación de tabletas digitales en las aulas. De los 8 centros primeramente escogidos y contactados de Cataluña, 3 descartaron la posibilidad de participar en la investigación. Finalmente, participaron en el estudio un total de 5 centros educativos: 2 de educación primaria, 1 de secundaria y 2 escuelas-instituto. Se realizaron 15 entrevistas, en las cuales han participado un total de 21 entrevistados. Se entrevistaron por separado a los 3 grupos de cada centro: dirección, coordinación TIC y familias.

\begin{tabular}{l|ll|l|ll|l} 
& Primaria & & Secundaria & Primaria y Secundaria & \\
\hline Colectivo & CENTRO 1 & CENTRO 2 & CENTRO 3 & CENTRO 4 & CENTRO 5 & Total \\
\hline Equipo directivo & 1 & 1 & 2 & 3 & 1 & 8 \\
Coordinador/a TIC & 1 & 1 & 1 & 1 & 1 & 5 \\
Familias & 2 & 1 & 1 & 2 & 2 & 8 \\
\hline Total & 4 & 3 & 4 & 6 & 4 & $\mathbf{2 1}$
\end{tabular}

Tabla 1. Número de entrevistados según centro y colectivo.

Las entrevistas se realizaron entre mayo y junio de 2015 y duraron entre 15 y 40 minutos. Fueron registradas con una grabadora de voz y transcritas literalmente. Para analizar su contenido, se siguieron los siguientes pasos: 
1. Síntesis de las ideas con unidades de significado. Se extrajeron las ideas relevantes de cada entrevista a través de unidades de significado. Cada unidad de significado es una palabra o una frase breve que representa uno o más fragmentos de las entrevistas con la misma idea.

2. Categorización. Se realizó una tabla de doble entrada comparativa para cada colectivo (equipos directivos, coordinadores TIC y familias). En cada una de las tres tablas, se clasificaron las unidades de significado según el centro educativo y según las categorías que se habían establecido para las entrevistas (motivo de la incorporación, aplicación, coordinación entre niveles educativos, gestión de las tabletas en el centro, ventajas, problemas y limitaciones). Este sistema facilitó tener una visión clara de las diferentes ideas que habían surgido en las entrevistas para proceder a la descripción.

3. Descripción y representación. Una vez obtenidas las tablas comparativas, se pudieron contrastar las diferentes ideas de cada colectivo a través de descripciones. Paralelamente, las ideas más relevantes fueron representadas en tablas, las cuales se muestran en el apartado de resultados.

\section{RESULTADOS}

A continuación, se presentan a través de tablas los resultados de las entrevistas realizadas a los equipos directivos, coordinadores TIC y familias.

\subsection{Equipos directivos}

Motivos de la incorporación

- Por las características funcionales: portabilidad y apps.

- Tiene menor coste que los portátiles.

- Información por parte de la fundación Trams.

- Experiencia con el plan 1x1 en ordenadores portátiles.

\section{Aplicación}

E.P.

- Los centros compran las tabletas.

- Número reducido.

- Uso para primaria, infantil y todo e centro.

- Escuelas concertadas: iPad

- Escuelas públicas: tableta Android.

\section{Gestión de la tableta}

E.P.

\section{ESO}

- Se acaba el plan $1 \times 1$ con portátiles.

- Los alumnos en 19 de ESO

el tableta.

- Escuelas concertadas: iPad

- Instituto público: tableta Android.
Ventajas

- Es portable e inmediata.

- Es multiuso.

- Ocupa menos que un netbook.

Fácil de conectar a la red.

- Las apps muchas tareas
- Forma parte del material de la escuela.

- Se almacenan en un lugar fijo.

- Hay una tabla horaria para reservarlas o están distribuidas por las aulas.

\section{Coordinación entre niveles educativos}

- Hay continuidad con el uso del dispositivo.

- La manera de utilizarlo es diferente entre las dos etapas.

\section{Formación de los docentes}

- Se recibe formación: a nivel interno o externo.

\section{ESO}

- Cada alumno es responsable de su tableta.

- Deben cumplir una normativa de uso.

\section{Limitaciones}

- El teclado de la pantalla es menos confortable que el del ordenador problemas

Problemas

- Puntualmente
pueden surgir
problemas de
conectividad.


- Se comienza formando a un pequeño grupo de docentes y el resto se va añadiendo.

Tabla 2. Resultados del análisis de las entrevistas a los equipos docentes

Los centros educativos participantes, tanto en las etapas de primaria como de secundaria, habían tenido experiencia previa con el uso de ordenadores y, ante la llegada de las tabletas digitales, se plantean cambiar el dispositivo por temas funcionales. La tableta es más portable, permite descargar muchas aplicaciones de manera instantánea y tiene menor coste que los ordenadores.

Los centros se preocupan para que los docentes tengan una formación adecuada antes y durante la implementación de este dispositivo. Se realizan formaciones de manera interna, entre el personal de la misma escuela, o externamente, con profesionales que vienen a impartir cursos o a través de charlas a otras entidades.

En secundaria se implanta un modelo de $1 x 1 \mathrm{y}$, en primaria, mayoritariamente, es el mismo centro que adquiere un número reducido de tabletas para poder compartir entre toda la escuela. En secundaria son los alumnos quienes se hacen cargo de comprar la tableta y de responsabilizarse de llevarla cargada cada día y mantenerla con memoria suficiente. Además, deben cumplir una normativa de buen uso y son sancionados si no lo hacen.

En educación primaria la escuela se encarga de las tabletas digitales como parte del material escolar. Generalmente están guardadas en un carro -aunque no siempre se utiliza para transportarlas- situado en un lugar fijo del centro. En este espacio, los alumnos y maestros pueden ir a buscar las tabletas, guardarlas, enchufarlas a los cargadores y reservarlas en la tabla horaria. Hay espacios al horario que están reservados permanentemente para determinadas áreas y cursos, pero las otras horas libres se pueden reservar con antelación.

El centro 1 es el único que sigue el modelo que dispone en el aula unas tabletas fijas de apoyo. En cada aula de educación infantil y ciclo inicial se dispone de 4 tabletas digitales guardadas en una caja. De esta manera, tanto los alumnos como los maestros pueden acceder a las tabletas en cualquier momento, aunque haya pocas. Las tabletas se utilizan como rincón cuando los alumnos finalizan las tareas de clase y las pueden coger de manera autónoma, sin tener que salir de la clase.

Como excepción, el centro 5 es el único de los participantes que implementa un plan 1x1 en la etapa de educación primaria, concretamente en ciclo superior $y$, por tanto, se implementa y se utiliza de la misma manera que en secundaria.

\subsection{Coordinadores/as tic}

\begin{tabular}{ll}
\hline Aplicación & Ventajas \\
- Se pretende disponer de más tabletas. & - No conllevan problemas técnicos notables. \\
- Se pretende continuar proponiendo iniciativas & - Funcionan rápido y de inmediato. \\
con las tabletas y las TIC. & - Hay mucha variedad de apps funcionales. \\
- Las aulas y el centro están equipados con más & - Aparato intuitivo. \\
aparatos TIC: proyectores, PDI, ordenadores,... & - Los alumnos están habituados a hacer uso de \\
- Se daban más problemas técnicos los primeros & dispositivos móviles. \\
meses de implementación. & - Facilidad para conectar a la red.
\end{tabular}




\section{Gestión de las tabletas}

- El coordinador TIC organiza los horarios para disponer de las tabletas propias del centro.

- El centro elige el modelo de tabl

las familias del adquieran (ESO).

Es altamente portable y pesa poco.

- Sistema operativo y funcionamiento sencillo.

- Tiene un procesador suficiente para realizar las tareas escolares.

Mantenimiento Limitaciones

- Las familias deben contratar un seguro para la - Depende del modelo de tableta escogido puede dar tableta de los hijos (ESO). más o menos problemas.

- Los alumnos no pueden descargar apps que - El claustro debe estar preparado para afrontar la no sean para uso escolar. incorporación de las TIC.

- Las tabletas no requieren demasiado - Hay que tener criterio para elegir en qué momento mantenimiento. hay que utilizar la tableta, otro dispositivo o ninguno. - La metodología no viene dada por el aparato.

cargarán cada día.

- Se deben limpiar las pantallas.

- Se debe mantener la tableta con espacio de almacenamiento

\section{Formación de los docentes}

\section{Problemas}

- Los coordinadores y / o el equipo de TIC - El teclado del ordenador es más cómodo. apoyan y promocionan actividades al resto del - Es un dispositivo frágil y delicado. profesorado.

- El coordinador TIC es un referente.

- El mismo perfil de alumno problemático es quien puede hacer un mal uso del dispositivo.

- Hay docentes que son más reacios que otros. - Hay poca competencia digital docente.

- Se descubren las potencialidades de la tableta - Se dan pocos problemas técnicos, se puede bloquear antes de instaurar la foto. o desconectar

Tabla 3. Resultados del análisis de las entrevistas a los/las coordinadores/asTIC

Como afirman los coordinadores TIC (ver tabla 3), en comparación con un ordenador portátil, la batería de las tabletas dura mucho más. Es un aparato pequeño y ligero, por lo que no es necesario estar en un lugar fijo con el dispositivo enchufado y teniendo que apoyarse en una superficie plana. El teclado de los ordenadores se considera más cómodo cuando se tiene que escribir, por esta razón, se permite a los alumnos que lleven teclados para tableta.

No requiere demasiado mantenimiento: recargar la batería cada día, mantener espacio de almacenamiento interno y limpiar la pantalla. En la ESO se hacen cargo los propios alumnos y, con respecto a las tabletas propias del centro, se determina un encargado, que puede ser un maestro o el coordinador TIC. El coordinador TIC gestiona la disponibilidad de las tabletas del centro, a través de una tabla horaria donde se anotan las horas en que los dispositivos están reservados y para qué cursos y áreas. No se dan problemas técnicos notables, puede ocurrir que alguna tableta se desconecte de la WiFi o que se bloquee, pero los problemas se daban con más frecuencia en los primeros meses de implementación. Por este motivo, los coordinadores TIC recomiendan que la infraestructura de conectividad esté bien asegurada antes de instaurar un número elevado de tabletas digitales u otro dispositivo que requiera conexión a Internet. Dos de los centros participantes, para asegurar una buena atención ante problemas técnicos que puedan darse en la escuela, contratan a un profesional que se hace cargo. 


\subsection{Familias}

Conocimientos sobre el uso educativo de la Ventajas

\section{tableta}

- Se utiliza como libro digital.

- Se utiliza para buscar información.

- Uso de plataformas virtuales.

- Jugar a apps.

- Es una herramienta como lo es el ordenador.

- Se pueden realizar videoconferencias.

- Es una herramienta de trabajo.

\section{Gestión de la tableta de los centros}

- En algunos centros no se informa a las familias sobre el uso educativo de la tableta en las aulas.

- En algunos centros sí se informa a las familias sobre el uso educativo de la tableta en las aulas.

- Los centros establecen una normativa que los alumnos deben cumplir.

\section{Preocupaciones de las familias}

- Necesidad para conocer funciones de la tableta.

- Se tiene desconocimiento de las redes sociales.

- Miedo a que los alumnos accedan a contenidos inapropiados.

- Se pretende que alumnos no vean la tableta como una herramienta exclusiva de juego.

- Las familias deberían tener formación tanto de las tabletas como de otros aspectos de la educación de los hijos.

- Las familias deberían conocer sobre las TIC para guiar a sus hijos.

- Se debe tener cuidado con la información personal que se comparte.

\section{Tabla 4. Resultados del análisis de las entrevistas a las familias}

Lo que principalmente preocupa a las familias y que es el motivo por el que están de acuerdo en utilizar la tableta a nivel educativo, es que sus hijos ven la tableta como un juego. En cambio, la percepción de uno de los familiares es diferente, dado que primero el alumno ha conocido el dispositivo en la escuela, para trabajar, y luego en casa: "En nuestra casa ha pasado, el iPad ha llegado una vez a la escuela ya estaba. Por lo tanto, es una herramienta de trabajo. No es una herramienta lúdica. Hay lúdico? Claro que sí. Pero es una herramienta de trabajo" (Familiar 1, centro 4).

El uso de las redes sociales preocupa porque es fácil que los alumnos comuniquen información personal y las familias tienen mucho desconocimiento sobre este tema, por lo que están de acuerdo en que necesitarían información por parte del centro sobre cómo guiar a sus hijos. Las familias ven necesario que los centros las puedan asesorar o poder ofrecer formación de algún tipo sobre el uso de las redes sociales y otros temas de las TIC que ya que se teme porque se desconoce la potencialidad de la herramienta, como afirman algunos entrevistados. 
Las familias tienen un conocimiento de las TIC a nivel de usuario, sobre las herramientas y dispositivos que utilizan en su día a día tanto en casa como en el trabajo, pero desconocen la potencialidad educativa que tienen las tabletas. Como algún centro lo hace, las familias están agradecidas cuando se realiza algún tipo de jornada informativa sobre el uso que hacen sus hijos en la escuela y el instituto de las tabletas.

En general, las familias están satisfechas con que los alumnos aprendan con las TIC y con la tableta digital ya que ven que es una herramienta que ocupa un papel importante en la vida cotidiana y que es primordial que desde la escuela se trabaje con estos dispositivos, de cara al futuro profesional de los alumnos.

\section{DISCUSIÓN Y CONCLUSIONES}

\subsection{Finalidades con el uso de tabletas digitales}

Teniendo en cuenta el modelo SAMR (Romrell et al., 2014), los centros ya tienen experiencia con las TIC y la tableta se incorpora para substituir los ordenadores portátiles, dado que es un dispositivo más económico. En cada centro la tableta se adquiere, inicialmente, para realizar las mismas tareas que se realizaban con los ordenadores aunque, con la experiencia, los centros afirman que ofrece más ventajas que los anteriores dispositivos.

Del mismo modo con el que se utilizaban los portátiles se han empezado a utilizar las tabletas en primaria y en secundaria: en primaria, cuando la tableta es propiedad del centro y no hay dispositivos para todos los alumnos, para un uso más ocasional y estipulado en algunas actividades; en secundaria para un uso más transversal y como herramienta cotidiana de trabajo, ya que cada alumno tiene su dispositivo.

Las tabletas, igual que los portátiles en su momento, se adquieren para unas determinadas finalidades pedagógicas que son diferentes en cada centro y etapa educativa. Ante esta variedad, se apunta a que la integración de las TIC debe ser progresiva en los distintos momentos de la vida escolar y se debe contemplar el contexto en el que se envuelven (Camarda, 2012).

\subsection{Aspectos técnicos}

Para integrar las TIC en los centros educativos es primordial garantizar las competencias docentes en la integración curricular de las mismas (Carneiro et al., 2011), con la finalidad de asegurar que los maestros y profesores están habilitados para utilizar las herramientas digitales.

También, para la incorporación de un número considerable de nuevos dispositivos tecnológicos, es indispensable asegurar una buena conexión a la red. Una vez las tabletas están en funcionamiento, el mantenimiento es muy sencillo ya que es un aparato que tiene un software fácil de manejar. Cuando las tabletas son propiedad del centro, es el equipo educativo quien se encarga del mantenimiento como un material más en la escuela.

En cambio, en un plan de $1 \times 1$, son los alumnos quienes se deben preocupar de esta tarea. No se pueden descargar el contenido que quieran, sino que se utiliza 
exclusivamente con fines educativos y los centros establecen una normativa de buen uso, de modo que si un alumno accede a contenido inapropiado, es sancionado. Los alumnos ven la tableta como una herramienta de trabajo y el perfil de alumno que se distrae y hace un uso inapropiado, es el mismo alumno que sin tableta también lo hace.

Ante el desconocimiento de la potencialidad educativa de esta herramienta, entre las familias se generan miedos y prejuicios ya que la tableta se considera por muchos usuarios una herramienta de ocio y entretenimiento (UNESCO, 2012). Por este motivo, las familias consideran que es importante que estén asesoradas sobre la implementación de la tableta digital. Se pueden realizar charlas a los padres y madres $\mathrm{u}$ ofrecer una jornada de puertas abiertas para que se pueda ver como los alumnos trabajan con las tabletas, como lo propone uno de los centros participantes.

\subsection{Aspectos organizativos}

Los equipos docentes se forman progresivamente ya que hay maestros y profesores que son más reticentes a la hora de hacer uso de las tecnologías. Los docentes más implicados en el uso de las TIC son quienes asisten primero a las formaciones externas y organizan formaciones internas a nivel de centro. En todos los casos, hay docentes de referencia que promocionan el uso de la tableta digital y ofrecen apoyo al resto del equipo, normalmente es el coordinador TIC y otros maestros implicados. Para una buena organización en la formación de los docentes sobre el uso de las TIC, hay que identificar a las personas que tienen iniciativa para innovar y liderar proyectos (Lugo \& Kelly, 2011).

En la etapa de educación primaria se puede apreciar diversidad en cuanto al modelo de organización de tabletas digitales. Solamente en la educación primaria las tabletas son propiedad del centro y generalmente se opta por disponer de un lugar fijo de almacenamiento. Un maestro determinado o el coordinador TIC, se hace cargo de gestionar el horario para reservas y del mantenimiento de los dispositivos. En las aulas de ciclo inicial de uno de los centros se dispone de cinco tabletas para los rincones. En este caso, los dispositivos están en la misma aula y los mismos alumnos las pueden coger sin tener que salir fuera. En ciclo superior de uno de los centros y en todos los centros de educación secundaria se implementa un modelo de 1x1 y las tabletas son propiedad de los alumnos. Estos modelos de implementación se incluyen entre los que propone Marquès (2013). No se puede considerar ningún modelo como más válido que otro, sino que en cada situación se opta por una manera de distribuir las tabletas u otra, según las necesidades educativas y también según el presupuesto del centro o de las familias.

\subsection{Conclusiones generales}

Como conclusión, se establece que los centros tienen en cuenta los mismos aspectos técnicos y organizativos para integrar tabletas digitales: experiencia con las TIC, formación docente, infraestructura de conectividad, mantenimiento, organización para su uso en las aulas e información para las familias.

Las diferencias y semejanzas en el momento de tratar estos aspectos técnicos y organizativos no radican entre etapas educativas, sino más bien a nivel de modelo de 
distribución de tabletas: entre un plan de $1 \times 1$ y los modelos en los que los dispositivos son propiedad del centro.

Para futuras investigaciones, se propone evaluar diferentes modelos de distribución de tabletas dentro de la misma etapa educativa, con la finalidad de establecer cuáles son más apropiados en cada caso.

\section{REFERENCIAS}

BALANSKAT, A. (2013). Introducción de las tabletas en los centros educativos: Evaluación del proyecto piloto para tabletas Acer y European Schoolnet. European Schoolnet. Recuperado a partir de http://recursostic.educacion.es/eda/web/tabletas/documentos/TabletEvaluatio n_exec-summ_ES.pdf

BISQUERRA, R. (2009). Metodología de la investigación educativa. (La Muralla, Ed.) (2. ed.).

CAMARDA, P. (2012). Manual general introductorio. ( $1^{\text {a }}$ edición). con la colaboración de María Gabriela Madeo ... [et.al.]: Buenos Aires: Ministerio de Educación de la Nación. Recuperado a partir de http://portales.educacion.gov.ar/primariadigital/files/2014/04/manual_primari a_digital_aulas_digitales_moviles.pdf

CANALES, M. (2006). Metodologías de Investigación Social. ( $1^{\text {a }}$ edición). Santiago, Chile: Lom Ediciones.

CARNEIRO, R., TOSCANO, J. C., \& DÍAZ, T. (2011). Los desafíos de las TIC para el cambio educativo. La educación que queremos para la generación de los Bicentenarios. (Organización de Estados Iberoamericanos (OEI), Ed.)Los desafíos de las TIC para el cambio educativo (Metas educ). Fundación Santillana. Recuperado a partir de http://www.oei.es/publicaciones/detalle_publicacion.php?id=10

CATALDI, Z., \& LAGE, F. J. (2013). Entornos personalizados de aprendizaje (EPA) para dispositivos móviles: situaciones de aprendizaje y evaluación. Revista de Educación Mediática y TIC, 2(1), 117-148. Recuperado a partir de http://www.edmetic.es/Documentos/Vol2Num1-2013/7.pdf

Consell Superior d'Avaluació del Sistema Educatiu (2010). El projecte eduCAT1x1. Una aproximació en la perspectiva de les directores i directors de centres participants (curs 2009-2010). Generalitat de Catalunya. Departament d'Educació. Recuperado a partir de http://csda.gencat.cat/web/.content/home/consell_superior_d_avalua/pdf_i_a Itres/static_file/eduCAT1x1.pdf

Consorci d'Educació de Barceloma (2013). L'ús de les TIC als centres EduCAT 1x1 de la ciutat de Barcelona. Generalitat de Catalunya. Ajuntament de Barcelona. Recuperado a partir de http://www.edubcn.cat/rcs_gene/extra/00_tecnologia_educativa/informe_us_ tic_centres_1x1.pdf

FORNELL, R., \& VIVANCOS, J. (2010). El Pla TAC de centre. - (Col-lecció TAC; 1) 
(Generalita). Servei de Tecnologies per a l'Aprenentatge i el Coneixement Edició. Servei de Comunicació i Publicacions. Recuperado a partir de http://www.bcn.cat/imeb/pec/forum_exit/Pla_Tac_de_centre.pdf

HEDBERG, J. G. (2008). Education design research - Edited by J van den Akker. British Journal of Educational Technology, 39(3), 559-560. http://doi.org/10.1111/j.1467-8535.2008.00855_1.x

LUGO, M. T., \& KELLY, V. (2011). El modelo 1 a 1: un compromiso por la calidad y la igualdad educativas. La gestión de las TIC en la escuela secundaria: nuevos formatos institucionales. Organización de las Naciones Unidas para la Educación, la Ciencia y la Cultura. Instituto Internacional de Planeamiento de la Educación. Buenos Aires. Recuperado a partir de http://observatorio.relpe.org/wpcontent/uploads/2012/02/lugokelly_compromisocalidaded_conectar.pdf

MARQUÈS, P. (2013). Las tabletas digitales en las aulas de clase: 4 modelos tecnológicos para facilitar su utilización. Recuperado 1 de septiembre de 2015, a partir de http://peremarques.net/tabletasaulas.htm

Mobile World Capital Barcelona (2013). El manual de l'Expert. mSchools. Recuperado a partir de http://experts.mobileworldcapital.com/files/experts-mschoolsintro.pdf

PUENTEDURA, R. (2010). SAMR and TPCK: intro to advanced practice. Retrieved February. Recuperado a partir de http://hippasus.com/resources/sweden2010/SAMR_TPCK_IntroToAdvancedPra ctice.pdf

ROMRELL, D., KIDDER, L. C., \& WOOD, E. (2014). The SAMR model as a framework for evaluating mLearning. Journal of Asynchronous Learning Network, 18(2), 1-15.

SPRADEY, J. P. (1980). Participant Observation. United States of America: Holt, Rinehart and Winston.

UNESCO (2012). Activando el aprendizaje móvil en América Latina. Iniciativas ilustrativas e implicaciones políticas. París: Organización de las Naciones Unidas para la Educación, la Ciencia y la Cultura. Recuperado a partir de http://unesdoc.unesco.org/images/0021/002160/216080s.pdf

VACCHIERI, A. (2013). Estado del arte sobre la gestión de las políticas de integración de computadoras y dispositivos móviles en los sistemas educativos (Programa T). Argentina: Fondo de las Naciones Unidas para la Infancia (UNICEF). Recuperado a partir de http://www.unicef.org/argentina/spanish/edudacion_Estado_arte_gestion_poli ticas.pdf 


\section{Para citar este articulo:}

Fernández, L. \& Carrera, X. (2016). Aspectos organizativos y técnicos para la integración de tabletas digitales en centros de educación primaria y secundaria de cataluña. EDUTEC, Revista Electrónica de Tecnología Educativa, 58. Recuperado el $\mathrm{dd} / \mathrm{mm} /$ aa de http://www.edutec.es/revista 